\title{
Filigrane
}

Écoutes psychanalytiques

\section{Entre vents et marées ou la psychanalyse en dépit des formes et des formules}

\section{Jean-Charles Crombez}

Volume 23, numéro 2, automne 2014

Qu'est la psychanalyse devenue ? Partie 2

URI : https://id.erudit.org/iderudit/1028920ar

DOI : https://doi.org/10.7202/1028920ar

Aller au sommaire du numéro

\section{Éditeur(s)}

Revue Santé mentale au Québec

ISSN

1192-1412 (imprimé)

1911-4656 (numérique)

Découvrir la revue

Citer cet article

Crombez, J.-C. (2014). Entre vents et marées ou la psychanalyse en dépit des formes et des formules. Filigrane, 23(2), 13-26.

https://doi.org/10.7202/1028920ar

\section{Résumé de l'article}

La psychanalyse est un dispositif de mise en scène d'un sujet. D'une part, la psychanalyse est un dispositif de mise en conscience et de mise en lien. Malgré les empreintes de ses origines qui la supportent et la freinent, elle soutient un processus hors des temps chronologiques, des espaces culturels et des pathologies spécifiques. D'autre part, la psychanalyse est un instrument de mise en question et de mise en jeu. Elle interroge et relance les réalités et leurs sens, les vécus et leurs origines, les crises et leurs fonctions. Du même souffle, ses théories et ses pratiques sont mises à l'épreuve, en dégageant les premières du risque de conservatisme et en préservant les deuxièmes du danger de dispersion. Et puis, la psychanalyse est un outil de création, de mise en oeuvre. Elle place deux sujets là où tout peut s'avérer nouveau et imprévu. Elle peut apporter à tous les thérapeutes, au-delà des différents modèles thérapeutiques, une lucidité et une liberté dans l'exercice de leur fonction. 




\title{
Entre vents et marées ou la psychanalyse en dépit des formes et des formules ${ }^{1}$
}

\author{
Jean-Charles Crombez
}

\begin{abstract}
La psychanalyse est un dispositif de mise en scène d'un sujet. D'une part, la psychanalyse est un dispositif de mise en conscience et de mise en lien. Malgré les empreintes de ses origines qui la supportent et la freinent, elle soutient un processus hors des temps chronologiques, des espaces culturels et des pathologies spécifiques. D'autre part, la psychanalyse est un instrument de mise en question et de mise en jeu. Elle interroge et relance les réalités et leurs sens, les vécus et leurs origines, les crises et leurs fonctions. Du même souffle, ses théories et ses pratiques sont mises à l'épreuve, en dégageant les premières du risque de conservatisme et en préservant les deuxièmes du danger de dispersion. Et puis, la psychanalyse est un outil de création, de mise en oeuvre. Elle place deux sujets là où tout peut s'avérer nouveau et imprévu. Elle peut apporter à tous les thérapeutes, au-delà des différents modèles thérapeutiques, une lucidité et une liberté dans l'exercice de leur fonction.
\end{abstract}

\section{Une rencontre...}

'est l'histoire d'une rencontre fortuite. Cette personne sort d'un ascenseur un matin. C'est une patiente; elle vient d'avoir son congé du service de psychiatrie et se dirige vers la sortie de l'hôpital. Elle est habillée pauvrement et porte dans ses deux sacs de poubelle tous ses effets personnels. J'étais en train d'attendre cet ascenseur pour y monter, presqu'impatient à cause d'un retard dans mon horaire. Elle me reconnaît! Je dis bonjour par convention, prêt à pénétrer dans cet ascenseur, prêt à échapper à ce tête-àtête. Mais elle m'interpelle et commence à parler: c'est donc la rencontre d'une patiente et d'un impatient!

Je vais décrire cette rencontre selon plusieurs niveaux, un peu comme ces anciennes planches d'anatomie où l'on pouvait découvrir plusieurs couches en soulevant des morceaux du dessin qui montraient tour à tour les muscles, les organes, les vaisseaux, puis le squelette: de la surface à la structure.

Ainsi, pour un observateur à distance, il y a ce couloir, ces gens qui attendent, sortent et entrent continuellement dans ces ascenseurs. Et il y a 
cette personne portant sur son dos ses sacs de vêtements. Et puis, il y a moi, immobilisé, la regardant. Pas de mouvement, nous sommes tous les deux debout. Il y a finalement le moment, un peu plus tard, où elle sourit et s'en va vers la sortie alors que je me prépare à prendre le prochain ascenseur.

En étant plus proche, ce témoin aurait accédé à l'entendu: deuxième niveau de ce qui s'est passé. Elle parle de son éjection, un congé imposé qui la laisse démunie. Elle parle de ses douleurs, de sa précarité, de son désarroi, de son désespoir. Elle est perdue et ne sait où elle va aller. Moi, je ne dis rien, enfin pas vraiment: des sons, des interjections, des monosyllabes,... Elle continue à parler, pleure un peu, me fait part, par bribes, de ce qu'elle vit. Au bout de dix minutes, elle s'arrête, et me dit: Je me sens mieux, je peux partir, je vous remercie.

Le troisième niveau sera le thème de cet écrit. C'est la face cachée de l'événement, sous ce que l'on voit, sous ce que l'on entend. Les questions qui se posent portent sur ce qui s'est passé dans cette rencontre, le changement qui s'y est produit, le rapport entre cette rencontre et ce changement. Et elles soulèvent aussi la nature de cette rencontre, son effet thérapeutique et son dispositif psychanalytique.

\section{Une psychothérapie?}

Mais d'abord: peut-on parler de thérapie, d'un acte thérapeutique? D’autant que la psychothérapie a été récemment légiférée au Québec. Sa définition et sa description ont même été enchâssées dans les règlements édités dans la Gazette officielle (2012). Il y est d'emblée précisé que la psychothérapie est le traitement d'un trouble mental, d'une perturbation comportementale, ou de tout autre problème; et qu'elle se dispense selon quatre modalités: psycho-dynamique, cognitivo-comportementale, systémique-communicationnelle ou existentielle-humaniste. Donc la première question qui se pose est de savoir si la rencontre d'un malade qui souffre et qui s'adresse à un professionnel en vue d'un soulagement, la classe d'emblée comme psychothérapeutique? La deuxième question, en miroir: est-ce que l'intervenant qui reçoit et accepte une telle demande, dans une intention thérapeutique qu'il partage du fait-même, se positionnerait ainsi comme psychothérapeute? La troisième question, s'il s'agit bien de psychothérapie reconnue, devrait préciser quel modèle a été utilisé dans cette rencontre, puisque seuls certains d'entre eux sont admis comme valables ${ }^{2}$; ou plusieurs modèles sont-ils en cause; ou tous ces modèles à la fois? Et le psychothérapeute, maintenant autorisé par le malade, a-t-il eu toutes les formations 
dans ces différents modèles pour en assurer la validité et sa légitimité? Et puis, il y a une dernière question, plus fondamentale: si, au décours de la rencontre il y a eu changement, dans ce trouble mental, cette perturbation comportementale ou tout autre problème, peut-on déduire, après coup, qu'il y a eu psychothérapie? Sinon, y aurait-il des changements possibles en dehors des psychothérapies conformes? À voir ce qui se passe actuellement dans la chasse aux pratiques devenues illégales, ces dernières questions ne sont nullement hypothétiques bien que totalement absurdes.

Il faut dire que cette loi définit aussi ce que n'est pas la psychothérapie, et y précise qu'on ne doit pas considérer comme tels les interventions de soutien, les rencontres d'accompagnement, les coachings qui visent l'accentuation de potentiel, les interventions de crise, etc. Ceci est fort intéressant, car ces différentes approches ont des résultats aussi communs et connus qu'indéniables sur les troubles mentaux, les perturbations comportementales et d'autres problèmes. Plus encore, ces approches non-psychothérapeutiques n'y sont pas définies selon des modèles, mais selon leurs fonctions. Or, il est justement reconnu que la logique et l'utilisation-même de ces modèles ne sont pas significatifs en ce qui concerne leur efficacité dans les changements obtenus; ceci pose la question de leur utilité spécifique. En ce sens, il a été prouvé que certains facteurs non spécifiques, sous-jacents non seulement aux approches psychothérapeutiques reconnues, mais également aux approches définies comme non psychothérapeutiques, se révèlent d'une efficacité majeure (Lecomte, Savard, 2004).

D'ailleurs il faut rappeler un problème épistémologique: tout modèle, quel qu'il soit, n'est qu'une approximation d'une réalité autrement plus complexe, en l'occurrence ici celle de l'humain, d'un humain. On ne peut y réduire personne; on ne peut donc penser l'appliquer à tous sans exception et sans nuance, hors d'une ambitieuse illusion de savoir et de pouvoir. Tenant compte de cette contrainte de la réalité, il serait important de comprendre et de permettre le fonctionnement des facteurs dits non spécifiques, en-deçà de tout modèle à appliquer et de toute technique à suivre.

\section{Une psychanalyse?}

Si ce qui s'est passé dans cette rencontre ne peut pas, ou ne doit pas, être nommé psychothérapie, pourrait-il s'agir de psychanalyse? S'il ne peut évidemment pas s'agir d'une séance de psychanalyse, pourrait-il s'agir d'un travail psychanalytique? En effet, la psychanalyse s'est, souvent et justement, distanciée de tout dessein thérapeutique au sens d'une intervention axée sur 
la disparition de symptômes, c'est-à-dire d'un traitement, pour se proposer d'abord comme un lieu de compréhension d'une personne.

Si j'osais proposer une définition actuelle d'une psychanalyse, propre à ma vision et à mon élan, il s'agirait d'un processus de connaissance. Et ce, dans trois acceptions de ce terme: celui d'une ouverture de conscience, celui d'une éclosion pour ne pas dire d'un accouchement, et celui d'une rencontre qui est le dispositif de cette ouverture et de cette éclosion. Je la définirais comme telle pour échapper à la catégorisation des distinctions théoriques de cette conscience, des multiples aspects de cette éclosion et des diverses modalités de cette rencontre.

En-deçà des recherches originaires et originales, avec leurs fulgurantes intuitions et leurs questionnables affirmations, et au delà des dérives nombreuses, parfois décriées, et des altercations majeures, et de leurs scissions subséquentes (Roudinesco, 1982, 1986), en-deçà et au-delà donc, je veux voir dans la psychanalyse la formidable mise en scène d'un sujet, de sa vie intérieure, de sa pensée et de son histoire, de sa mémoire et de son désir, le tout essentiellement humain, mystérieux, apparemment illogique et fondamentalement personnel.

Le sujet se construit dans un cadre d'émotion, de pensée, de vie, défini par la famille et la culture. Son adhésion à ces systèmes est nécessaire à sa survie quotidienne, et cette obligation forge des dynamiques dont il devient prisonnier. Alors le sujet se retrouve perdu, affaissé ou éclaté. Le travail d'analyse est de permettre à ce sujet d'advenir, à travers les murs de la logique, de la convenance et de la certitude. Il s'agira parfois de le retrouver, oublié qu'il a été quelque part, parfois de le trouver, interdit qu'il a été, donc nulle part. Il s'agira d'en rassembler les morceaux clivés, refoulés, réprimés, cachés dans les zones sombres et ombrageuses de l'inconscient.

Il y est essentiellement question de vie et de mort, de fidélité et de désir. Les symptômes, les conflits, seront les voies d'entrée de cette retrouvaille et de cette trouvaille; ce sont des voies d'entrée, par occasion ou par prétexte, mais ils n'en seront pas la cible réelle. Tout symptôme et tout conflit sont des cadavres: c'est pour cela qu'on peut les analyser et les découper à l'infini et à satiété. Les symptômes et les conflits sont du mort, le problème est ailleurs, partout ailleurs, toujours ailleurs: ailleurs dans l'espace, ailleurs dans le temps, et ailleurs dans les personnes. C'est la trouvaille du sens qui en retrace l'origine, elle, vivante, et qui permet de s'en dégager en la replaçant dans l'histoire du sujet. Il y a cette fable du gars qui cherche en pleine nuit sa clef sous un réverbère. Et puis, s'invite gentiment quelqu'un pour l'y aider, sans plus de 
résultat; car la clef est ailleurs, là-bas dans l'ombre, là où il l'a perdue. Alors, pourquoi cherche-t-il sous ce réverbère? Parce que dans la lumière, il est plus facile de chercher! C'est ainsi que la connaissance en psychanalyse n'est pas dans l'évidence, mais dans ce qui échappe à la preuve, dans ce qui n'est ni prouvable, ni prouvé. Le psychanalyste travaille dans l'ombre, dans la non-évidence, dans ce qui n'est pas explicable, dans ce que l'explication ne résout pas.

Il est possible de prendre tout ceci comme un truisme et une banalité. Mais il y a là quelques conséquences fabuleuses. L'une est que chaque problème rapporté sera le levier d'une ouverture de conscience personnelle, d'une transformation, d'un passage. Ensuite, chaque transformation appellera et impliquera celle de tous, de toutes les figures et de tous les événements passés, présents et futurs. Au lieu d'une élimination de ce qui importune, à la manière d'un traitement (médical ou psychothérapeutique, par exemple), dorénavant, on proposera un accès à ce qui est incompris, à ce qui n'a pas été compris, à ce qui n'a pas été tenu en compte, à ce qui n'a pas été inclus. On va passer du local évident au global latent... tout simplement!

\section{Le cadre institutionnel}

Peut-on dire que l'espace et le temps dans lesquels s'est déroulée cette rencontre ont permis une démarche psychanalytique? Pour avancer dans la réponse à cette question, il faut faire quelques remarques préliminaires sur le cadre de cette rencontre particulière.

Le mode de cette rencontre, en-deçà de la situation bizarre dans un couloir, est très spécifique. D'une part, elle est bien celle de deux personnes dans un cadre professionnel, et non pas dans un contexte social ou amoureux. D'autre part, cette rencontre professionnelle n'est pas de nature administrative, ou d'intention médicale d'évaluation, de prise en charge ou de traitement. Elle est bien celle de deux personnes, joignant la demande de l'une d'elle et la présence de l'autre, et de ce qui s'y joue sans aucun autre outillage que cette relation.

Cette rencontre fait place aussi, pour ma part, à la conjonction de deux désirs: celui de travailler auprès de gens hospitalisés et considérés comme malades, et celui d'y inscrire des rencontres personnelles afin que les personnes ne soient pas réduites à leur statut de malade. Comment peuvent alors cohabiter les cadres psychanalytique et institutionnel, comment permettre du relationnel dans de l'institutionnel?

Le cadre psychanalytique classique est celui d'un lieu protégé des bruits, des incursions, des occupations. Ce lieu réservé, écarté de la réalité extérieure, 
vise à favoriser les manifestations de la réalité intérieure, psychique, personnelle et insoupçonnée. Or le cadre institutionnel est fondamentalement différent: ouvert à toute injonction. La personne qui y entre se voit dépossédée de son univers familier, de ses biens, de ses atours, de ses habitudes, de ses vêtements. Elle y devient non protégée, vulnérable. Nous dirions que, si la maladie l'a submergée et l'a rendue malade par son invasion incontrôlable, l'institution qu'on nomme paradoxalement hospitalière, ne fait qu'ajouter l'insulte à l'injure quant à cette invasion qu'on a pu dire barbare (Arcand, 2003). Certes l'idée est de délivrer la personne, devenue patiente, de cette maladie importante; mais le prix à payer est lourd par la perte d'identité qu'elle impose ou qu'elle met en péril. Le mouvement de dépersonnalisation y est donc inverse de celui de la psychanalyse: une perte de sujet versus un gain de sujet. Cela ressemble à un curieux contrat faustien: je te promets la survie si tu me donnes ta vie!

Nous parlons d'hôpitaux, mais cette dynamique de dépersonnalisation et de dépossession existe dans la dynamique de toute institution. Elle se fait en général sous prétexte de contrôler un problème actuel ou futur, et à la condition que la personne donne en gage une partie d'elle-même pour bénéficier de cette aide demandée. Il s'agit ici de maladie, mais on peut tout autant parler du règlement d'un conflit, d'un manque d'argent, d'une perte: ce sont des institutions judiciaires, financières ou compensatoires. Ceci est bien exprimé par l'adage au sujet des banques: elles ne désirent que notre bien.

Cependant il est des lieux institutionnels qui préservent l'identité des personnes. On connaît par ci par là des exemples d'unités psychiatriques animées par d'anciens patients, des villages thérapeutiques dans plusieurs pays, des communautés de prises en charge, des maisons de soins palliatifs ou gériatriques... Quelques tentatives de s'attarder davantage à la personne ont aussi été développées: un clown par ci, un silence par là, quelques ballons, quelques chants, de l'art ou du mouvement. Ces initiatives sont parfois conséquentes d'une réflexion humaniste. Mais elles peuvent aussi résulter d'une observation factuelle: en effet, la perte du sujet en milieu institutionnel entraîne des complications quant à l'efficacité des traitements, la compliance à ceux-ci et l'apparition de maladies nouvelles. Que de symptômes iatrogéniques et de résistances diverses surviennent alors! 


\section{Un processus thérapeutique}

Cela m'a donc amené à penser, voire tenter la psychanalyse dans ce cadre institutionnel et à porter le regard sur ce qui se déroule grâce au dispositif analytique, sous-jacent aux formes qui en sont les véhicules. Dans cette optique, sous les différentes modalités de l'exercice de la psychanalyse, il y a un processus thérapeutique3. Ce processus de transformation, de croissance et de compréhension est naturel, intelligent, complexe, global et passablement non conscient. Il est à l'image des processus de guérison et de création, mis au défi par différents évènements et obstacles, limité comme eux et posant alors la nécessité de l'apport d'une aide, de l'intervention d'un tiers. Ce processus appartient à tout le monde, du moins on l'espère encore. Il est d'intérêt public et ne devrait pas être l'objet d'un usage réservé ou exclusif.

Cette réflexion m'a poussé à chercher ce qui était fondamental en analyse, afin de l'extraire des formes classiques qui ne pouvaient être utilisées dans le contexte hospitalier — pas de divan, pas de fauteuil, pas d'intimité privée, pas de rencontres régulières, pas de séances à durée prévue, une position debout auprès du lit ou assis sur son bord, avec des personnes intubées - , et de commettre ce fondamental dans la pratique quotidienne et les actes de soins, afin que ces gestes pratiques témoignent aussi d'un lieu analytique.

Ce qui nous importe, au-delà des systèmes et des techniques psychanalytiques, c'est une retrouvaille de l'être, en deçà des symptômes et des conflits. D'une part, l'adresse de la rencontre ne sera pas ceux-ci, comme dans le cas d'un traitement, mais la personne elle-même dans sa souffrance. Il s'agira d'animer ce processus qui sera alors le moteur de la régression éventuelle des symptômes et de la résolution des conflits. On rencontre donc la personne, tout simplement et tout difficilement.

D'autre part cette transformation ne se fait pas par l'action de l'un sur l'autre, ce qui est de l'ordre des traitements, qu'ils soient médicaux ou cognitifs, mais par la rencontre de l'un avec l'autre. Le processus a pour théâtre cette rencontre, et le psychanalyste y est profondément impliqué (Elkaïm, 2001). Mais comment? Ce qui la caractérise, c'est l'entre deux: entre deux du relationnel et de l'institutionnel, entre l'immobilité apparente des deux personnages et le brouhaha qui l'entoure.

En deçà des pratiques très régulées et des théories très sophistiquées, je cherchais les aspects de la rencontre et les composantes du dispositif permettant la libération du processus psychanalytique entravé. Donc, ici, nous ne penserons nullement la psychanalyse en tant que théorie, mais à 
la lumière des conditions dans lesquelles elle peut se découvrir et s'exercer. En fait, ce sont des conditions simples, mais, curieusement, pas très faciles à soutenir, et assez faciles à ignorer. Elles tournent autour de la reconnaissance de la personne comme être particulier, de celle d'un non-conscient pourtant fort présent et portant tous les temps de la signature des liens, de l'histoire et du désir: le futur-présent et le passé-présent.

Ces conditions incluent aussi la relation que permet le psychanalyste, la «danse» qu'il propose et le parcours personnel que permet cette danse (Crombez, 2013). Au fait, en tant que c'est une danse, il faut avoir appris à danser! Il faut en effet savoir danser le tango avant d'y inviter un partenaire sans lui marcher sur les pieds, et cela ne s'apprend pas qu'en lisant les livres de psychanalyse! C'est simple: la psychanalyse ne peut s'exercer sans un parcours personnel préalable, alors que les interventions de traitement ne le nécessitent nullement4.

\section{Points de repère pour une intervention psychanalytique}

Dans une relation psychanalytique, outre les facteurs dits non spécifiques, il y a des points de repère, et je vais en dégager cinq. Ce ne sont surtout pas des critères de temps et d'espace, et cela n'a directement rien à voir avec le nombre de rencontres, le lieu des séances, la durée de ces rencontres, le type d'aménagement de ce lieu. Ce que nous allons dégager, ce ne sont donc pas des éléments formels, mais des positions essentielles. Si ces éléments formels, par exemple le nombre de séances admissible, ont probablement été indispensables au développement de la psychanalyse, cette réalité historique risque de perdurer sous la forme de règlements anachroniques, et la psychanalyse pourrait bien s'enfermer dans les formes qui l'ont vu naître. Confinée ainsi dans des modèles anciens, elle se livrerait par là-même à son extinction. Il nous semble donc important de souligner ces points de repère fondamentaux au-delà desquels certaines variantes notamment du cadre sont possibles.

Ces positions essentielles ont la qualité et le défaut d'être fort simples, tout au moins à première vue. La qualité d'être simples, parce qu'ils permettent de mettre en place un dispositif analytique dans la plupart des situations et de tenter de le faire dans toutes. Le défaut d'être simples, parce qu'on peut les déconsidérer par leur banalité («tant d'études pour cela, et tant de réflexions pour en arriver là, voyons donc!»). Fort simples aussi à première vue, mais au demeurant fort difficiles à mettre en œuvre, et à tenir. 


\section{A. L'accueil}

On pourrait considérer l'accueil comme évident, tellement qu'il ne vaudrait même pas la peine d'en parler. En psychanalyse, on en fait trop souvent peu de cas, et il est surtout la marque de commerce des thérapies humanistes. N'empêche, l'accueil de la personne en tant que telle, au-delà de sa maladie ou/et de son caractère, témoigne d'un lieu où tout pourra se dire, sans que rien ne soit retenu contre la personne. Tout y sera valide et validé: les réalités et les fantasmes, en deçà de classifications diagnostiques ou de catégorisations pathologiques. Cette position de validation n'a pas du tout de valeur morale; elle procède d'une logique. En effet, le but de la démarche n'est pas l'élimination de quoi que ce soit, mais le mouvement qui en advient. Il est donc nécessaire d'accueillir les rêves dans tous les sens du terme: l'imaginaire et les désirs, les rêves passés et futurs. L'attention flottante portera tout autant sur les lapsus, les actes manqués, les incertitudes, les affects, les souvenirs et les projets. Tout est possiblement sensé.

C'est une mise en conscience qui retrouve la personne effacée, sa pensée interdite, ses rêves anéantis. Elle confronte l'individu, réduit à ses comportements, ses symptômes, ses étiquettes, ses consommations, ses règlements et ses contrats. Ce premier geste, l'accueil, en est un de reconnaissance des masques, de ce qui masque, de ce qui obstrue et de ce qui occulte la conscience, de ce qui empêche le pleur et le cri. C'est une reconnaissance de la personne en tant que sujet, des événements en tant que relatifs, et des vécus intérieurs en tant que fondamentaux.

\section{B. Le lien}

La deuxième composante du dispositif psychanalytique est le lien. Celui-ci a revêtu différents visages au cours des décennies : l'association libre, le transfert, le lien primaire, l'attachement, l'abréaction... Sous ces formes variées se démontre une évidence profonde: tout est relié, pour le meilleur et pour le pire. Tantôt des carences de liens et des coupures de liens; tantôt des assuétudes et des asservissements aux liens. Le passé retient la personne par des besoins inassouvis et la lie par ses conflits de loyauté (DucommunNagy, 2010) et ses contrats faustiens (Lefebvre, 1984). Le présent reprend le passé par ses transferts incontournables; le futur nie le présent dans ses contraintes ambitieuses, que l'on dira grandioses et narcissiques.

Dès lors tout un travail de mise en lien se construira autour de ce tissage à la fois nécessaire à la vie et risquant tout autant de l'étouffer: un travail de liaisons, de reliaisons et de déliaisons. Ce sont ces liens qui feront sens, 
qui permettront de retrouver ce sens perdu. Ainsi, les catégorisations, les exclusions, les hiérarchisations, les relations de cause à effet, ne seront pas de mise: elles éloignent le sens en l'enfermant, en l'étiquetant. Ce dévoilement des liens, cette ouverture du sens aura fonction de liberté. Une liberté par rapport à l'histoire propre et à celle des autres, de quelque génération soientils. La mise en lien autorisera la levée des secrets et des contraintes pour une libération intérieure.

\section{Le questionnement}

La troisième composante est le questionnement. C'est-à-dire que tout est remis en question: les affirmations et les faits, les souvenirs et les fantaisies, les émotions et les affects, les souvenirs et les expectatives. Cette mise en question est au cœur de l'amorce d'un travail psychanalytique en milieu hospitalier.

Le malade, y compris ses symptômes mentaux, y est souvent considéré sous le versant biologique. Et toutes les informations, tous les examens et traitements, sont arrimés à ce modèle causaliste et matérialiste. Il faut donc y accoler, puis y introduire, une dimension historique et culturelle: c'est le questionnement qui en est le plus souvent la clef. Celui-ci peut être présent dès le début des rencontres, par une conscience aiguë, parfois préoccupante depuis longtemps pour le patient, qu'il serait, pour ainsi dire, la cause de sa maladie. Alors cette conscience s'entoure trop souvent d'une atmosphère de culpabilité déconcertante ou d'une combativité obsessive. Parfois, c'est plutôt l'épuisement des ressources de la médecine et les complications des traitements qui font que le patient, ou son soignant, se résigne à tenir compte de la complexité de la réalité et de l'insuffisance des affirmations antérieures. Le regard se déplace alors, quand l'écoute et le lien ont été établis, de l'éradication espérée du mal, désormais mise en échec, à l'extinction de soi évoquée par la personne.

Qu'est-ce donc que cette maladie, que ce symptôme, que ce mal? La psychosomatique commence là: il n'y a pas que des causes, mais aussi des origines, des contextes, des facilitateurs, des terrains. Bref, au plus profond, le patient pourrait bien y être pour quelque chose. Et les maladies pourront être comprises comme des organisations figées et inhabitées: "le corps de personne» (Crombez, 1980). Ce questionnement prend forme, tout simplement, dans un échange au pied du lit, au bord de la personne. Le reste suit, la porte s'ouvre, et le regard s'éclaire. Ce questionnement n'est pas fait pour savoir, pour colliger de quelconques informations; il est là pour étonner, 
pour permettre. Il s'agit avant tout d'interroger des certitudes, des a priori, des enseignements, des théories. Il s'agit de défaire des constructions figées pour libérer des processus.

\section{Le jeu}

Avec le jeu, on aborde un lieu moins fréquenté par la psychanalyse hors des pratiques avec les enfants (Winnicott, 1975) et des méthodes psychodramatiques (Crombez, 2009). Il s'agira d'une mise en jeu du corps, d'une reprise de corps.

Il est important de l'aborder ici au regard du contexte institutionnel. En effet, la réflexion et le questionnement ne suffisent pas à redonner une place à la personne, au vu de toute l'agitation de la pratique hospitalière. Je parle tout autant des multiples gestes médicaux que des déplacements dans des corridors vers des salles d'examens, de multiples scopies et de la chaise musicale des changements de lit et de chambre.

Devant ce brouhaha extérieur, il faut mettre en place une action intérieure. Celle-ci sera gage d'une éventuelle maîtrise des événements divers. Ce corps soumis qui est patient, cette personne insoumise qui est impatiente, on les met en rapport. Puisque l'on a pu penser le corps, que peut-on maintenant en faire? Projet fou sous l'œil du raisonnement, expérience étonnante par la sensation vivante qu'elle procure. On met donc en jeu la maladie et les traitements dans un univers intérieur, comme le ferait l'enfant dans son bac à sable (Crombez, 2007). Un psychodrame intérieur dont les acteurs sont les parties du corps, les morceaux d'histoires et les bribes de symptômes. Ce qui parait fou devient l'évidence. Et cela se fait au chevet de la personne hospitalisée ou dans le décours d'une rencontre de suivi. Dès que la mise en scène est installée, par tous les ingrédients précédents que sont la mise en conscience, la mise en lien et la mise en question, l'action, c'est-à-dire le jeu, peut commencer. Comme on le lance lors d'un tournage: «action!».

\section{E. La création}

La cinquième composante est celle de la création, elle qui nous rapprochera de l'originalité de la psychanalyse au delà de l'accueil humaniste, de la liaison systémique, du questionnement cognitif ou psychodynamique, et du jeu psychodramatique. C'est cette création qui me semble au cœur de la psychanalyse en tant que processus particulier.

On a tout fait, on a tout dit, on a tout compris, et survient un moment crucial, celui où le processus psychanalytique prend toute sa singularité, et la 
relation psychanalytique toute sa nécessité. Il n'y a soudain plus rien à dire, ni à imaginer, ni à comprendre, et ce, de part et d'autre, du côté de l'analysant et du côté du psychanalyste. Une immense ignorance et une immense impuissance se répand: l'analysant ne sait plus quoi penser, et le psychanalyste quoi dire. C'est là un moment de vérité et un lieu de bascule. C'est là où le regard va changer; cet instant, longtemps évité et foncièrement désiré, à l'insu de notre bon vouloir.

Ce moment, tous le vivent profondément, parfois constamment, parfois brièvement; chacun en souffre, sans pouvoir le partager nulle part ni avec personne, tant la souffrance en est profonde et indicible. Cette ignorance et cette impuissance, les personnes les rencontrent dans leur vie, au hasard d'événements inexprimables, de ruptures d'habitudes, de pertes de certitudes. Pourtant, les échappées sont multiples hors de cette expérience d'impasse, de doute et de solitude.

C'est dans ce lieu que la relation psychanalytique prend toute sa valeur grâce à son dispositif ouvert. Cette zone est un passage délicat, source de pertes d'identité, de rappels de vulnérabilité. C'est le défi du psychanalyste, de la psychanalyse que d'amener la personne vers ce passage, ce goulet, comme vers le col d'un sablier. C'est un lieu qui fera que tout sera différent après; c'est un lieu de version, de subversion. C'est aussi le lieu tant évité par le psychanalyste lui-même, celui où il pourrait perdre la tête, celui où même, parfois, il perd la tête. C'est pourtant celui où l'analysant pourra enfin perdre la tête pour retrouver son esprit (Crombez, 2008).

Dans les psychanalyses classiques, cela peut prendre du temps pour atteindre ce moment, vu les nombreuses occasions de s'en écarter. Il est néanmoins présent dès le début du travail psychanalytique, dans tous les silences de l'analysant et, on l'espère, dans le silence du psychanalyste. En effet, ce silence du psychanalyste ne saurait être une simple attente de la parole de l'autre; il indique d'abord que le psychanalyste se situe, dès le début, dans ce lieu et cette position où il est amplement ignorant et profondément impuissant. Il est ainsi déjà prêt à se situer, lorsque la personne y parviendra, dans cette zone qui signe la fin du connu et le début du commencement: une mise en oeuvre. Et le passage pourra se faire ici, par ce dispositif, sans la mort aiguë de la dépersonnalisation, et celle chronique de l'endormissement.

Or, dans l'hôpital, auprès des lits ou devant l'ascenseur, les personnes sont d'ores et déjà vulnérables. Elles ont perdu beaucoup: des capacités, des mémoires et des projets, auxquels s'ajoutent les douleurs et les contraintes. Cette zone d'ignorance et d'impuissance est toujours très proche, et c'est 
rapidement, sinon d'emblée, à cet endroit et à ce moment que se fait la rencontre potentiellement créatrice.

\section{En conclusion: un passage...}

Nous pouvons enfin aborder le troisième niveau de ce qui s'est passé devant cet ascenseur: celui-ci qui se passe en moi.

D'abord une tentative de fuite: "J'ai pas le temps, ce n'est pas la place!». Puis la mise en place d'un espace de rencontre, à l'intérieur de moi: «Bon, j'accepte d'écouter, de sentir et d'imaginer ». Puis ma position d'ignorance et d'impuissance, c'est-à-dire sans faire d'effort pour assurément comprendre ou absolument faire quelque chose. Finalement, laisser émerger un processus à travers cette rencontre.

C'est pourquoi je dirais que cette rencontre n'a pas été le lieu d'une psychothérapie dans le nouveau sens de ce terme, non plus le lieu d'application d'un modèle prévu, désigné ou respecté. Mais elle a été lieu d'analyse, très précisément, aussi brève et minuscule qu'elle ait pu être manifestement. Il n'y a certes pas eu d'analyse de transfert ou d'interprétation de conflits: ce n'était pas le temps! Mais il y a eu le fondement de tout processus psychanalytique: cette présence, cette rencontre pour elle-même, pour une rencontre avec soi-même, ce lieu d'expression de souffrance, ce passage à travers l'ignorance et l'impuissance.

«Je vais mieux, je peux aller, merci beaucoup.»

Jean-Charles Crombez jccrombez@approche-echo.net

\section{Notes}

1. Les lecteurs sont invités à consulter notre site web (www.revuefiligrane.ca) pour visionner la conférence de Jean-Charles Crombez, de laquelle est issu cet article.

2. Ainsi les modèles psychocorporel, ludo-artistique, ethnoculturel ou psychospirituel, pour ne citer que ceux-là, ne sont pas reconnus.

3. Il faut préciser qu'en utilisant le terme thérapeutique nous ne faisons plus référence à la psychothérapie, puisque celle-ci a été dorénavant définie comme un traitement particulier des maladies et problèmes mentaux. La psychothérapie n'a donc plus comme objet le processus thérapeutique. Ainsi la psychanalyse qui avait déjà peu à voir avec de la psychothérapie, s'en distingue désormais et m’apparaît toujours aussi compatible avec la conception d'un processus thérapeutique sous-jacent.

4. Dans cette logique, il n'est pas étonnant que les règlements québécois actuels de la formation à la psychothérapie, assimilée à un traitement, n'incluent nullement la nécessité d'une démarche personnelle qualifiée. 


\section{Références}

Arcand, D., 2003, Les invasions barbares, film franco-québécois.

Crombez, J.-C., 1980, Le corps de personne, Psychologie médicale, 12 (2).

Crombez, J.-C., 2007, La méthode en ECHO, Montréal, Éditions de l'Homme.

Crombez, J.-C., 2008, Solitude d'un thérapeute de brousse, Survivre, Colloque organisé par l'ETAP (Études et Traitements Analytiques par le Psychodrame), Paris, Société parisienne d'aide à la santé mentale.

Crombez, J.-C., 2009, Survivre à la vie: les aléas du changement, Changer, colloque organisé par l'ETAP (Études et Traitements Analytiques par le Psychodrame), Paris, Société parisienne d'aide à la santé mentale.

Crombez, J.-C., 2013, Entrez dans la danse, Jeux de transfert, colloque organisé par l'ETAP (Études et Traitements Analytiques par le Psychodrame), Paris, Société parisienne d'aide à la santé mentale.

Ducommun-Nagy, C., 2010 Loyautés familiales et processus thérapeutique, Cahiers critiques de thérapie familiale et de pratiques de réseaux, $\mathrm{n}^{\circ} 44,27-42$.

Elkaïm, M., 2001. Si tu m’aimes, ne m'aime pas, Approche systémique et psychothérapie, Paris, Seuil.

Lefebvre, P., 1984, La psyché de la somatose en quête de névrose: le sentier obstrué, Revue française de psychanalyse, $\mathrm{n}^{\circ} 5,1173-1182$.

Gazette officielle du Québec, 6 juin 2012, 144 année, n 23, 2893.

Lecomte, C., Savard, R., Drouin, M.-S., Guillon, V., 2004, Qui sont les thérapeutes efficaces? Implications pour la formation en psychologie, Revue québécoise de psychologie, 23 (3), 73-102.

Roudinesco, E., 1982, Histoire de la psychanalyse en France, vol. 1, Paris, Fayard, 1994.

Roudinesco, E., 1986, Histoire de la psychanalyse en France, vol. 2, Paris, Fayard, 1994.

Winnicott, D. W., 1975, Jeu et réalité, Paris, Gallimard. 